\title{
Poröse Poly-N-Isopropylacrylamid-Hydrogele für piezoresistive Sensoren mit schnellem Ansprechverhalten
}

\author{
Daniela Franke und Gerald Gerlach \\ Institut für Festkörperelektronik, Technische Universität Dresden, Dresden, Deutschland \\ Kontakt: daniela.franke@tu-dresden.de
}

\section{Einleitung}

Responsive Hydrogele sind quellbare Polymernetzwerke, die geeignet sind, auf die Änderung der Salz- oder Organikakonzentration, des $\mathrm{pH}$-Werts oder der Temperatur einer wässrigen Lösung mit einer Volumenänderung zu reagieren [1-4]. Sie sind somit für die Verwendung in Sensoren und Aktoren geeignet [5-9]. Ein Nachteil der Hydrogele ist der von der Probengeometrie abhängige, teils über Stunden dauernde Quellprozess, der die Nutzung in geregelten Messsystemen limitiert. Um dieser Einschränkung entgegenzuwirken und den Transport des flüssigen Mediums zu befördern, werden in dieser Arbeit mittels chemischer Synthese Poren als Kanalstrukturen in das temperatursensitive Hydrogel Poly- $\mathrm{N}$-Isopropylacrylamid (PNIPAAm) eingebracht. Versuche an einem porösen $\mathrm{pH}$ sensitiven Hydrogel zeigten bereits eine Verkürzung der Ansprechzeiten in einem piezoresistiven Sensor beim Wechsel zwischen verschiedenen $\mathrm{pH}-$ Werten [10].

In dieser Arbeit werden zwei poröse PNIPAAmHydrogele aus zwei verschiedenen Synthesemethoden bezüglich ihrer Porenstruktur charakterisiert sowie temperaturvariierte Messungen im piezoresistiven Sensor durchgeführt und mit einem unporösen PNIPAAmHydrogel verglichen. Zur Synthese beider poröser Hydrogele wird eine auf Tenside basierende Templatmethode verwendet. Für PNIPAAm 1 wird ein Tensid in einer Konzentration oberhalb der kritischen Mizellkonzentration in einer wässrigen Monomerlösung gelöst. Die sich bildenden kugelförmigen Mizellen, für die ein Durchmesser $<100 \mathrm{~nm}$ erwartet wird, werden als Poren im entstehenden Hydrogel abgebildet. Die die Mizellen enthaltende Templatlösung für die Synthese von PNIPAAm 2 wird um ein unpolares Lösungsmittel ergänzt, welches das unpolare Innere der Mizellen stabilisiert und zahlreiche weitere Aggregatstrukturen (lyotrope Flüssigkristallphasen) ermöglicht.

\section{Methoden und Materialien}

\section{Synthese der Hydrogele}

Für die Synthesen von unporösem und porösem, vernetztem PNIPAAm werden das Monomer $\mathrm{N}$ Isopropylacrylamid (NIPAAm), der Vernetzer $N, N^{\prime}$ Methylenbis(acrylamid) (MBA), das Redoxinitatorsystem Ammoniumpersulfat (APS) / N,N,N',N'Tetramethylethylendiamin (TEMED), die Tenside Brij ${ }^{\circ}$ L23 und AOT sowie das Lösungsmittel Heptan (alle Sigma-Aldrich, Germany) entsprechend der folgenden Synthesevorschriften verwendet.
Unporöses PNIPAAm: Eine Lösung aus $0.5 \mathrm{~g}$ (4.42 mmol) NIPAAm, $0.0068 \mathrm{~g} \quad$ (0.044 mmol) MBA, $26.3 \mu \mathrm{l}$ einer $0.84 \mathrm{M}$ APS-Lösung (enthält $0.02 \mathrm{mmol}$ APS), $3.33 \mu \mathrm{l}(0.02 \mathrm{mmol})$ TEMED und $3.5 \mathrm{ml}$ entionisiertem Wasser wurde in $1 \mathrm{ml}$-Glasröhrchen gefüll und zur vollständigen Polymerisation bei $4^{\circ} \mathrm{C}$ für drei Tage gelagert.

Poröses PNIPAAm 1: Zunächst werden $0.167 \mathrm{~g}$ (0.14 mmol) des Tensids BrijßL23 in $3 \mathrm{ml}$ Wasser gelöst, um eine klare mizellare Lösung zu erhalten. Darin werden $0.5 \mathrm{~g}$ (4.42 mmol) NIPAAm, 0,033 g (0.21 mmol) MBA und $0.0167 \mathrm{~g}(0.07 \mathrm{mmol})$ APS unter Rühren gelöst. Nach Zugabe von $7.5 \mu \mathrm{l}(0.05 \mathrm{mmol})$ TEMED wird die Lösung in $1 \mathrm{ml}$-Glasröhrchen gefüllt und über Nacht zum Polymerisieren stehen gelassen.

Poröses PNIPAAm 2: Zuerst wird eine wässrige Stammlösung bestehend aus $2.81 \mathrm{~g} \mathrm{(24.8} \mathrm{mmol)} \mathrm{NIPAAm,} 0.19 \mathrm{~g}$ $(1.2 \mathrm{mmol}) \mathrm{MBA}, 0.10 \mathrm{~g}(0.44 \mathrm{mmol})$ APS und $10 \mathrm{~g}$ Wasser hergestellt. Davon werden $3 \mathrm{~g}$ mit Heptan und AOT im Massenverhältnis Heptan : AOT: Stammlösung $=0.42$ : $0.42: 0.16$ zusammengegeben und gerührt bis eine klare Lösung entsteht. Zu dieser Lösung werden $63.5 \mu \mathrm{l}$ (0.42 mmol) TEMED gegeben. Nach kurzem Verrühren wird die Lösung in $1 \mathrm{ml}$-Glasröhrchen verteilt, in denen die Polymerisation über Nacht stattfindet.

Reinigung der Hydrogelproben: Die Hydrogelproben wurden nach der angegebenen Zeit aus den Glasröhrchen entfernt und für drei Tage (unporöses Hydrogel) bzw. 30 Tage (poröse Hydrogele) in entionisiertem Wasser gereinigt, welches täglich ausgetauscht wurde.

\section{Rasterelektronenmikroskopie (REM)}

Bruchstücke der oben beschriebenen zylindrischen Hydrogelproben wurden getrocknet, auf Kohlenstoffpads geklebt, mit Leitsilber kontaktiert und in der Sputteranlage Leica EM SCD500 der Firma Leica Microsystems $\mathrm{GmbH}$ (Wetzlar, Deutschland) mit $3 \mathrm{~nm}$ Platin beschichtet. Die REM-Bilder wurden im Gerät Neon 40EsB der Firma Carl Zeiss Microscopy GmbH (Jena, Deutschland) unter Verwendung einer Feldemissionskathode (Schottky-Emitter) bei EHT (Extra High Tension) von $3 \mathrm{kV}$ aufgenommen. Porendurchmesser wurden mit Hilfe der Software ImageJ [11] bestimmt.

\section{Sensormessungen}

Aufbau: Der verwendete Versuchsaufbau für die Sensormessungen wird schematisch in Abb. 1 a) gezeigt und wurde bereits in [5] und [10] beschrieben. 
a)

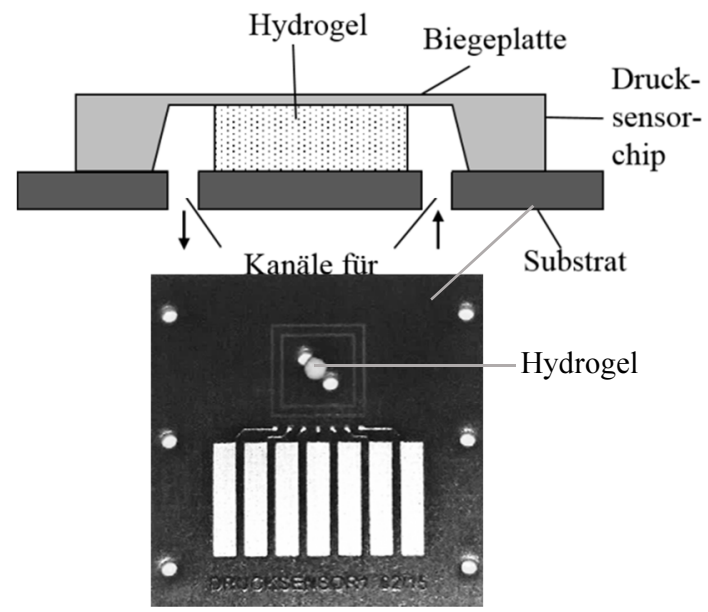

Abb. 1: Darstellung des piezoresistiven Sensoraufbaus

Ein zylindrisches Hydrogelprobenstück mit einem Durchmesser von $2.5 \mathrm{~mm}$ und einer Höhe von 1 bis $1.5 \mathrm{~mm}$ wird, wie in Abb. 1 a) abgebildet, auf eine Leiterplatte (hier als Substrat gekennzeichnet) zwischen zwei Bohrungen für den Wasserdurchfluss gelegt. Ein Drucksensorchip der C41-Serie der Firma Epcos (München, Deutschland) [12] wird so darüber positioniert und verklebt, dass sich die in Abb. 1 b) abgebildeten Kanalbohrungen sowie das Hydrogel innerhalb der Kavität des Chips befinden. Die Biegeplatte ist über dem gequollenen Hydrogelstück bereits vorausgelenkt. Über die Kanalbohrungen wird das Hydrogelstück von temperiertem Wasser umspült. Das resultierende Quellen und Entquellen des Hydrogels führt zu unterschiedlichen Auslenkungen der Biegeplatte. Daraus ergibt sich eine Änderung der Ausgangsspannung $V_{\text {out, }}$ welche mit Hilfe einer im Sensorchip integrierten Wheatstone-Brücke erfasst wird.

Sensormessungen: Über an der Unterseite der Leiterplatte angebrachte Schläuche zieht eine Pumpe (REGLO Digital MS-2/6) der Firma Ismatec (Wertheim, Deutschland) Wasser mit einer Fließrate von $7.2 \mathrm{ml} / \mathrm{min}$ durch den Sensorchip. Das Wasser wird außerhalb des Sensors auf zwei Heizplatten auf $25^{\circ} \mathrm{C}$ bzw. $50^{\circ} \mathrm{C}$ temperiert. Durch einen schnellen Wechsel der Heizplatten wird die Temperatur im Sensor abrupt geändert. Die Ausgangsspannung wird mit einem Fluke 45-Multimeter gemessen und mit dem Programm BIGA erfasst. Mit dem Netzteil Voltcraft Digi 35 wird eine Spannung von $5 \mathrm{~V}$ eingestellt. Vor Beginn der Messungen muss das getrocknete Hydrogel rehydriert werden. Dazu wird die Kavität des Sensors für mindestens 12 Stunden mit Wasser $\left(25^{\circ} \mathrm{C}\right)$ gespült. Die Messungen werden begonnen, wenn das Sensorsignal konstant ist.

\section{Ergebnisse}

Unporöses PNIPAAm: Die REM-Aufnahme in Abb. 2 a) zeigt, dass das unporöse PNIPAAm-Hydrogelstück keine Strukturierung im Volumen aufweist. Die Verteilung des Polymers in der Probe ist homogen.

a)

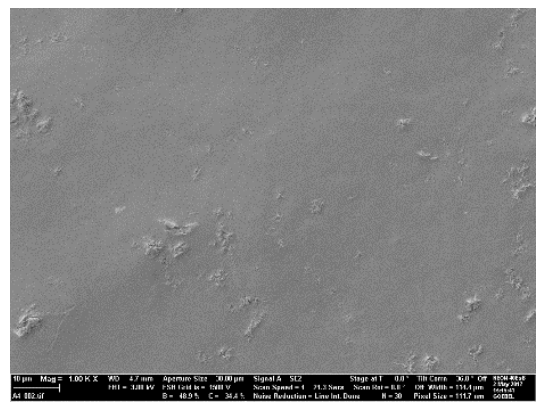

b)

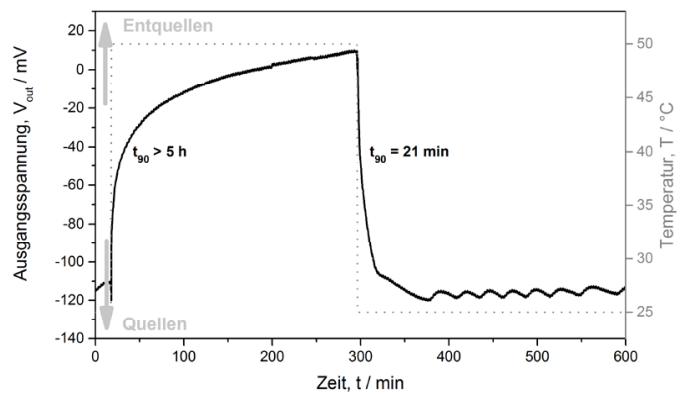

Abb. 2: REM-Aufnahme und temperaturabhängige Sensormessung - unporöses PNIPAAm

Abb. 2 b) zeigt die Sensormessung mit dem unporösen Hydrogel im piezoresistiven Aufbau bei zwei Temperatursprüngen $\left(25^{\circ} \mathrm{C}\right.$ zu $50^{\circ} \mathrm{C}$ und $50^{\circ} \mathrm{C}$ zu $\left.25^{\circ} \mathrm{C}\right)$. Im Diagramm ist auch dargestellt wie die Richtung des Messsignals mit dem Quell- und dem Entquellprozess korreliert. Die bereits angesprochene Problematik des sehr langsamen Quellverhaltens der unporösen Hydrogele verbunden mit einer langen Sensorantwort ist hier beim Sprung von $25^{\circ} \mathrm{C}$ zu $50^{\circ} \mathrm{C}$ deutlich zu erkennen. Nach einer Messzeit von ca. fünf Stunden wurde das Quellgleichgewicht bzgl. des Entquellens des Hydrogels nicht erreicht. Damit verbunden ist das Nichterreichen eines konstanten Sensorsignals. Für das Entquellen des Hydrogels im Sensor kann demzufolge keine Ansprechzeit t9o angegeben werden. Die in Abb. 2 b) angegebene Ansprechzeit für das Quellen (Temperatursprung $50^{\circ} \mathrm{C}$ zu $25^{\circ} \mathrm{C}$ ) des Hydrogels im Sensor ist sehr wahrscheinlich kleiner als der reale Wert, da zwar während der Messzeit ein konstantes Sensorsignal erreicht wird, das Quellen jedoch aus einem instationären Zustand heraus beginnt. Der Quellprozess ist demzufolge unvollständig. Es ist zu vermuten, dass ein stärker (bis zum Quellgleichgewicht) entquollenes Hydrogel mehr Zeit für die Quellung benötigt.

Poröses PNIPAAm 1: PNIPAAm 1 entsteht im Volumen makroporös wie Abb. 3 a) zeigt. Die Oberfläche ist synthesemethodenbedingt unporös, da Mizellen nur im Volumen der Syntheselösung vorliegen. Die Poren sind mitei- 
nander verbunden und weisen Durchmesser von $(3.8 \pm 2.1) \mu \mathrm{m}$ auf. Der Vergleich mit dem erwarteten Porendurchmesser von maximal $100 \mathrm{~nm}$ zeigt, dass sich während der Synthese kleine Mizellen zu größeren verbunden und zusammengelagert haben und somit größere Poren entstanden sind, die miteinander verbunden eine offene Kanalstruktur im PNIPAAm-Hydrogel bilden.

a)

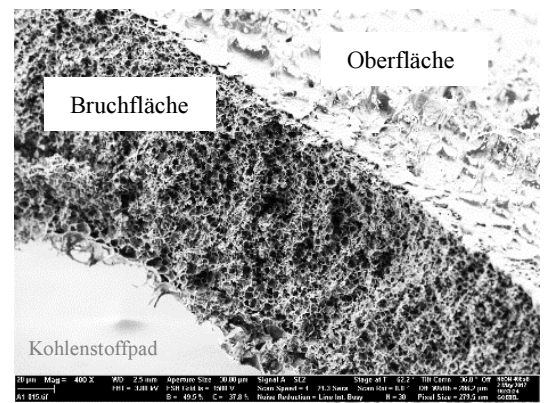

b)

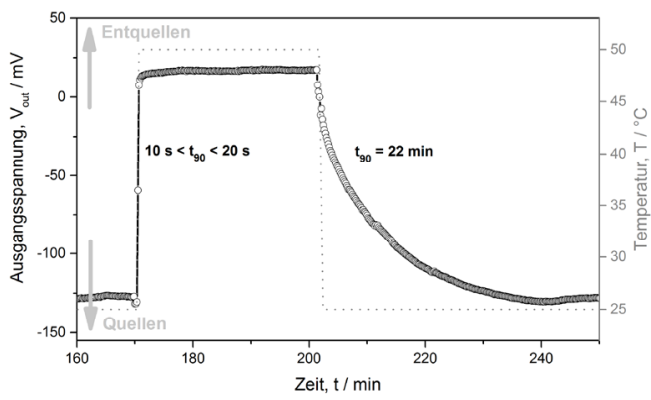

Abb. 3: REM-Aufnahme und temperaturabhängige Sensormessung - poröses PNIPAAm 1 (Die Bereiche Oberfläche und Bruchfläche des Hydrogelprobenstücks sind gekennzeichnet. Die Abtastrate der Sensormessung beträgt $1 / 10 \mathrm{~S} / \mathrm{s}$.)

Abb. 3 b) zeigt, dass die Ansprechzeit dieses Sensors auf eine sprunghafte Temperaturerhöhung $\left(25^{\circ} \mathrm{C} \mathrm{zu} 50^{\circ} \mathrm{C}\right)$ mit $<20 \mathrm{~s}$ deutlich kleiner ist als die für das unporöse PNIPAAm-Hydrogel in Abb. 2 b). Demgegenüber steht eine sehr lange Ansprechzeit von 22 min beim Temperatursprung von $50^{\circ} \mathrm{C}$ zurück zu $25^{\circ} \mathrm{C}$. Wir erklären diesen deutlichen Unterschied der beiden Ansprechzeiten damit, dass beim Prozess des Entquellens des Hydrogels die bereits ausgelenkte Biegeplatte des Drucksensorchips in Richtung ihres Ausgangszustands (keine Auslenkung) übergeht. Da dies ein freiwillig ablaufender Prozess ist, unterstützt die Rückstellung der Biegeplatte das Entquellen des porösen Hydrogels. Demgegenüber steht, dass das Quellen des Hydrogels eine Auslenkung der Biegeplatte verursachen muss. Da im porösen Hydrogel weniger quellbares Material pro Volumeneinheit verfügbar ist, kann angenommen werden, dass der Quelldruck des porösen Hydrogels im Vergleich zum unporösen kleiner ist. Die Auslenkungswirkung des porösen Hydrogels auf die Biegeplatte des Drucksensorchips ist entsprechend kleiner und führt zu einer längeren Ansprechzeit im Vergleich zum Entquellprozess.

Eine genauere Beschreibung und Diskussion dieses Sensors und des Sensorverhaltens ist in [13] aufgeführt.
Poröses PNIPAAm 2: Erste REM-Aufnahmen von der Oberfläche der PNIPAAm 2-Probe (Abb. 4 a) zeigen eine Strukturierung in Form zusammengelagerter Partikel mit Durchmessern von 100 bis 200 nm. Zwischen den Partikeln deutet sich Porosität in einer ähnlichen Größenordnung an.

a)

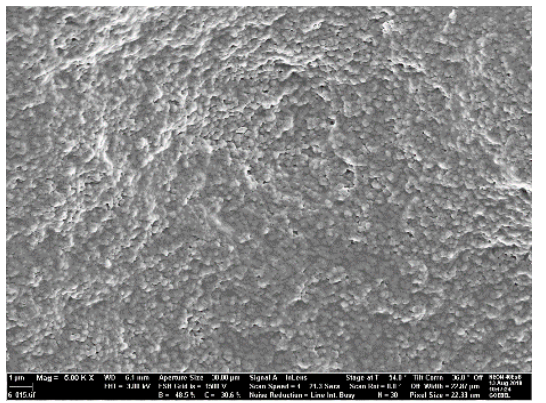

b)

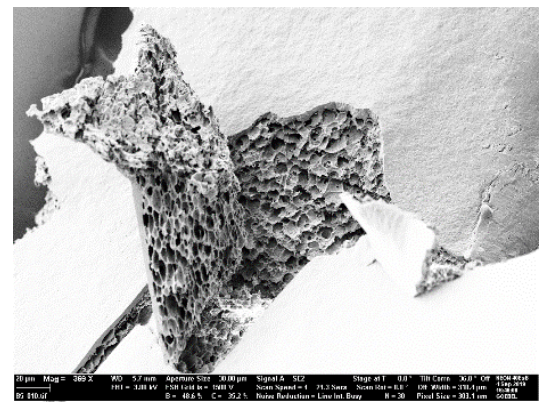

c)

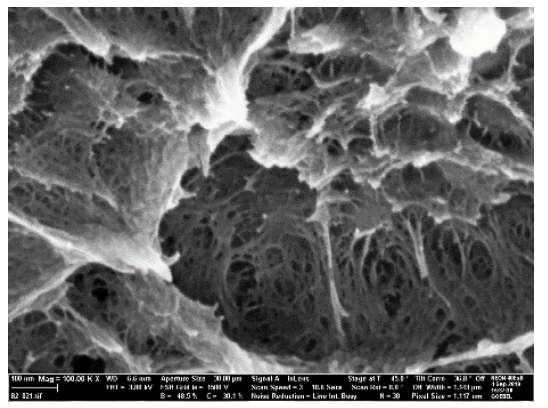

Abb. 4: REM-Aufnahmen - poröses PNIPAAm 2 (Bitte unterschiedliche Maßstäbe beachten!)

Durch zufällige Beschädigungen der Oberfläche, die durch die Vorbereitung der getrockneten Proben für die REM-Aufnahmen entstehen, ist die Porosität im Inneren der Probenstücke deutlicher zu erkennen (Abb. 4 b). Offenbar können mit der angewendeten Synthesemethode Poren mit Durchmessern im Bereich unter $100 \mathrm{~nm}$ entstehen (Abb. $4 \mathrm{c}$ ), was der angestrebten Größenordnung im Vergleich zu PNIPAAm 1 eher entspricht. Es sind jedoch weitere REM-Aufnahmen und eine angepasste, geeignete Probenpräparation notwendig, um die Porosität genauer zu charakterisieren.

Zudem sind die Sensormessungen noch Gegenstand aktueller Untersuchungen, weshalb an dieser Stelle noch kein Diagramm gezeigt werden kann. Freie Quellmessungen an Probenstücken vergleichbarer Dimension aus 
PNIPAAm 1 und PNIPAAm 2 deuten jedoch an, dass die Entquellzeit von PNIPAAm 2 um ein Drittel reduziert ist im Vergleich zu PNIPAAm 1. Dies ist gleichbedeutend mit einer Verkürzung der Entquellzeit mit Verkleinerung der Poren.

\section{Diskussion}

Vergleich des unporösen PNIPAAm mit PNIPAAm 1: Im Ergebnisteil wird dargestellt, dass das Einbringen von Porosität in das PNIPAAm-Hydrogel zu einer deutlichen Verkürzung der Quellzeit bis zum Erreichen des Quellgleichgewichts bei $25^{\circ} \mathrm{C}$ bzw. $50^{\circ} \mathrm{C}$ und damit auch zu einer verkürzten Ansprechzeit des Sensors führt. Dies ist damit zu erklären, dass die hier dargestellte offenporige Morphologie von PNIPAAm 1 einer Kanalstruktur gleichkommt, die dafür sorgt, dass das Wasser unabhängig von der temperaturabhängig wechselnden Hydrophilie und Hydrophobie des PNIPAAm aufgenommen bzw. abgegeben wird. Der Transport des Wassers im PNIPAAm-Hydrogel erfolgt somit nicht nur als Diffusion durch die Hydrogelstruktur (ein Prozess, der bei höheren Temperaturen aufgrund der Hydrophobie des PNIPAAm stark gehemmt ist), sondern auch konvektiv über die Kanalstruktur der Poren.

Vergleich PNIPAAm 1 und PNIPAAm 2: Wie im Ergebnisteil beschrieben deuten teils unveröffentlichte Ergebnisse an, dass PNIPAAm 2, welches kleinere Poren aufweist, schneller entquillt als PNIPAAm 1. Wir erklären dies damit, dass beim „Übergang“ von PNIPAAm $1 \mathrm{zu}$ PNIPAAm 2 die innere Oberfläche des Probenstücks, d.h. die spezifische Oberfläche des Materials, aufgrund der Verringerung des Porendurchmessers vergrößert wird. Äquivalent dazu würde eine großflächige Dünnschicht des PNIPAAm-Hydrogels schneller quellen und entquellen als ein kompaktes Hydrogelstück mit ähnlichem Volumen. Diese große innere Oberfläche erlaubt den großflächigen Transport des Wassers beim Temperaturwechsel in die bzw. aus der Hydrogelstruktur aus der bzw. in die oben beschriebene Kanalstruktur.

Die beschriebenen Diskussionspunkte lassen vermuten, dass die Sensorantwort unter Verwendung von PNIPAAm 2 beim sprunghaften Temperaturanstieg noch schneller erfolgt als mit PNIPAAm 1. Bezüglich der abrupten Temperatursenkung erwarten wir eine Verkürzung der Ansprechzeit, da mit der Vergrößerung der inneren Oberfläche der Hydrogelstücke eine Erhöhung der Anzahl der Porenwände pro Volumeneinheit einhergeht, die das Hydrogel gegen die Rückstellkraft der Biegeplatte stabilisieren sollte. Beides ist Gegenstand weiterer Untersuchungen.

\section{Literatur}

[1] Dušek, K. ; Patterson, D.: Transition in swollen polymer networks induced by intramolecular condensation. In: J Polymer Sci A-2: Polymer Phys 6 (1968), Nr. 7, S. 1209-1216

[2] Tanaka, T.: Collapse of gels and the critical endpoint. In: Phys Rev Lett 40 (1978), Nr. 12, S. 820-823
[3] Saito, S. ; Konno, M. ; Inomata, H.: Volume phase transition of N-alkylacrylamide gels. In: K. Dušek (Ed.) Responsive gels: volume transitions I. Springer, Berlin, 1993

[4] Orakdogen, N. ; Okay, O.: Reentrant conformation transition in poly (N,N-dimethylacrylamide) hydrogels in water-organic solvent mixtures. In: Polymer 47 (2006), Nr. 2, S. 561-568

[5] Gerlach, G. ; Arndt, K.-F. (Hg.): Hydrogel sensors and actuators, Kapitel 5 und 7, Springer, 2009

[6] Kuckling, D. ; Arndt, K.-F. ; Richter, A.: Temperature and $\mathrm{pH}$ dependent swelling behavior of poly $(\mathrm{N}$ isopropylacrylamide)-copolymer and their use in flow control. In: Macromol Mater Eng 288 (2003), Nr. 2, S. 144-151

[7] Guenther, M. ; Gerlach, G. ; Corten, C. ; Kuckling, D. ; Müller, M. ; Shi, Z. ; Sorber, J. ; Arndt, K.-F.: Application of polyelectrolytic temperature-responsive hydrogels in chemical sensors. In: Macromol Symp 254 (2007) S. 314-321

[8] Richter, A. ; Klatt, S. ; Paschew, G. ; Klenke, C.: Micropumps operated by swelling and shrinking of temperature-sensitive hydrogels. In: Lab Chip 9 (2009) S. 613618

[9] Richter, A. ; Paschew, G.: Optoelectrothermic control of highly integrated polymer-based MEMS applied in an artificial skin. In: Adv Mater 21, (2009) S. 979-983

[10] Schulz, V. ; Zschoche, S. ; Zhang, H. P. ; Voit, B. ; Gerlach, G.: Macroporous smart hydrogels for fastresponsive piezoresistive chemical microsensors. In: Procedia Engineering 25 (2011) S. 1141-1144

[11] https://imagej.net/ (abgerufen am 25.10.2019)

[12] Das Datenblatt kann nicht zur Verfügung gestellt werden, da das Modell vom Hersteller aus dem Programm genommen wurde.

[13] Franke, D. ; Binder, S. ; Gerlach, G.: Performance of fast-responsive, porous crosslinked Poly $(N$ isopropylacrylamide) in a piezoresistive microsensor. In: IEEE Sensors Letters 1 (2017), Nr. 6

\section{Danksagung}

Die Autoren bedanken sich bei M. Göbel vom Leibniz Institut für Polymerforschung Dresden (Dresden, Deutschland) für die Aufnahme der REM-Bilder. 